\title{
The Age Evolution of Ca II Emission in Late Type Stars
}

\author{
C. Trigilio ${ }^{1}$, S. Catalano ${ }^{2}$, E. Marilli ${ }^{3}$, V. Reglero ${ }^{4}$, \\ G. Umana ${ }^{1}$ \\ ${ }^{1}$ Istituto di Radioastronomia CNR, Stazione di Noto, C.P.169, \\ I 96017 Noto (SR), Italy \\ ${ }^{2}$ Istituto di Astronomia, Università di Catania, V.le A.Doria 6, \\ I 95125, Catania, Italy \\ ${ }^{3}$ Osservatorio Astrofisico, Città Universitaria, V.le A.Doria 6, \\ I 95125, Catania, Italy \\ ${ }^{4}$ Departamento de Matematica Applicada y Astronomia, Universidad de \\ Valencia, Spain
}

\section{Introduction}

It has been widely demonstrated that chromospheric emission is dependent on rotation rate (Hartmann and Noyes, 1987, Catalano, 1990, for recent reviews). Since the rotation decays with time, the chromospheric activity of single main sequence stars is also dependent on age. Catalano and Marilli (1983) suggested that the chromospheric emission of solar mass stars decays exponentially with the square root of the time, as recently confirmed by Barry (1988). Magnetic activity depends also on the convection parameters, i.e. on the mass. Since the evolution of surface rotation is faster for lower mass stars (Catalano et al., 1988), the internal rotation distribution, and therefore the dynamo action, should change with time in a different way for different masses.

In order to get insight into the evolution of dynamo action we have studied the CaII chromospheric emission as a function of mass and age. Here we present some preliminary results of observations of late type stars in young clusters and old moving groups.

\section{Observations}

The data we discuss refer to:

- photographic spectra obtained at La Silla ESO Observatory with the $1.5 \mathrm{~m}$ telescope coudé spectrograph (resolution from $0.45 \AA$ to $0.28 \AA$ );

- CCD spectra obtained at Calar Alto German-Spanish Astronomical Center with the $2.2 \mathrm{~m}$ telescope coudé spectrograph (resolution of $0.25 \AA$ );

- reticon spectra obtained at Calar Alto with the $1.23 \mathrm{~m}$ telescope Cassegrain spectrograph (resolution of $2.5 \AA$ ). 
The higher resolution has been used to observe 20 stars from F8V to K5V of low chromospheric emission belonging to old kinematic groups of solar age ( $\zeta$ Her, $\epsilon$ Indi, $\sigma$ Puppis, Arcturus group) or slowly rotating stars of the old disk population. Lower resolution has been used for the observation of 11 Hyades stars from $B-V=0.55$ to $B-V=0.9$ and 8 Pleiades stars from $B-V=0.7$ to $B-V=1.1$. Field stars of known high activity level have been observed at low resolution to test our extracted emission fluxes. All the spectra have been calibrated in absolute units at the stellar surface using the spectrophotometric atlas of Gunn and Stryker (1983) and the Barnes and Evans relation.

In addition to our $\mathrm{Ca}$ II emission, we have used data from the literature (i.e. from high resolution spectra, from Mt. Wilson spectrophotometer data and from $\mathrm{Mg}$ II $\mathrm{h}$ and $\mathrm{k}$ data converted to $\mathrm{Ca}$ II $\mathrm{H}$ and $\mathrm{K}$ luminosity). The Mt. Wilson data have been corrected for a revised minimum basal flux.

\section{Flux extraction}

The evaluation of the net chromospheric flux above the absorption profile has been done in different ways. For high resolution spectra of low emission stars we estimated the chromospheric flux as the difference between the observed spectra and the computed synthetic spectrum obtained from Kurucz (1979) RE model, using the SYNTH procedure (Castelli, 1987) convolved with a $0.35 \AA$ Gaussian for the cumulative effects of resolution, microturbulence and granulation.

For lower resolution spectra of active stars, the net chromospheric flux has been estimated from a direct comparison with spectra of old stars of similar spectral type convolved to reproduce the low resolution.

\section{Results}

The Ca II emission luminosity, for a given age, decreases smoothly with stellar mass, showing a knee at some critical mass. The knee moves from lower to higher masses as the age increases. It seems to disappear by the age of the Sun (Fig. 1).

The dependence of the $\mathrm{Ca}$ II luminosity from the mass can be fitted by power laws of the form

$$
L_{H K} \propto M^{\gamma}
$$

with different values of $\gamma$ for masses lower or higher than turnover point of the knee at a given age. For stars of $M \geq 0.8 M_{\odot}$ the emission can be represented as decaying exponentially with the square root of the age and with a rate increasing as the mass decreases (Fig. 2). At masses lower than the knee turnover value the decay rate of the emission seems to be independent of the mass, as already suggested by Catalano and Marilli (1983).

Acknowledgements. This work has been supported by the CNR, the M.U.R.S. through the University of Catania, the Osservatorio Astrofisico di Catania and the GNA(CNR). The extensive use of the computer facilities of the Catania ASTRONET site is also acknowledged. 


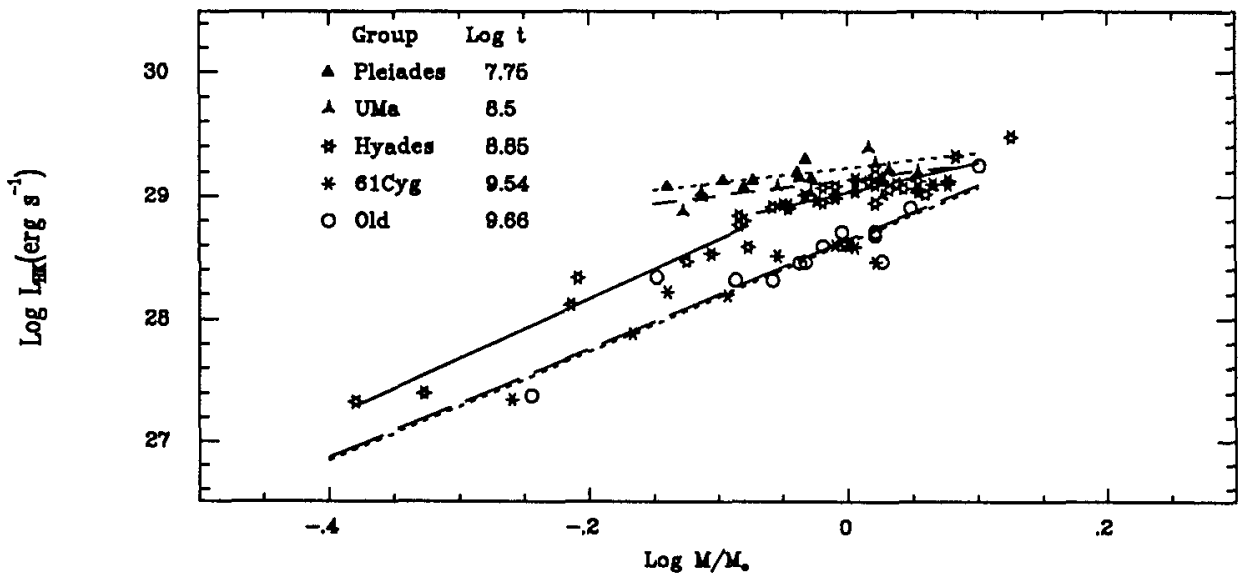

Fig. 1. $\mathrm{H}$ and $\mathrm{K}$ luminosity for MS stars of known age as a function of mass. Lines represent the fits to data for different groups of age and mass.

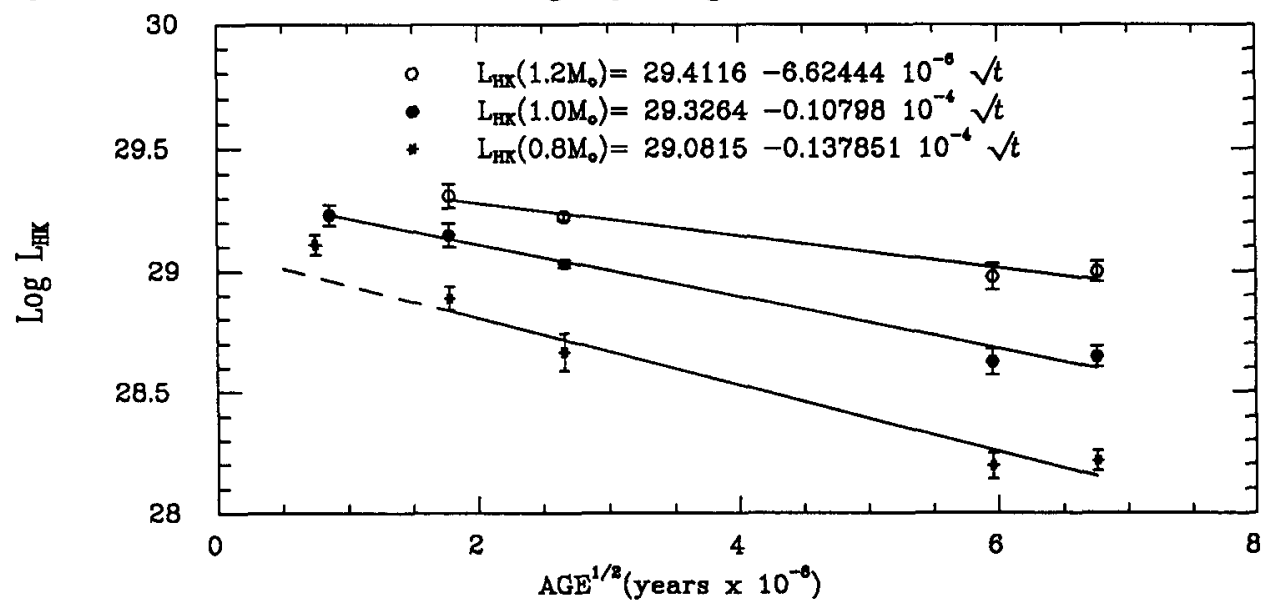

Fig. 2. Log of the $H$ and $K$ averaged luminosity versus square root of the age for three mass groups. A faster decay for low mass stars is apparent. Pleiades of $0.8 M_{\odot}$ do not seem to follow the decay rate of older stars.

\section{References}

Barry, D.C., 1987, Astrophys. J. 334, 436

Castelli, Publ. OAT., no. 984

Catalano S. and Marilli E.: 1983, Astron. Astrophys. 121, 190

Catalano S.: 1990, in NATO ASI on Active Close Binaries, eds. C. Ibanough and I. Yavez,

Kluwer Academic Publisher

Catalano S., Marilli E., Trigilio C.: 1988 in NATO ASI on Formation and Evolution of

Low Mass Stars, ed. A.K. Dupree, M.T.V. Lagoeds, Kluwer Academic Publishers

Gunn, J.E., Stryker, L.L.: 1983, Astrophys. J. Suppl. Ser. 52, 121

Hartmann, L.W., Noyes, R.W.: 1987, Ann. Rev. Astron. Astrophys. 25, 271

Kurucz R.L.: 1979, Astrophys. J. 40, 1 